\title{
Global cancer research initiative
}

This article was published in the following Dove Press journal:

Cancer Management and Research

30 April 2010

Number of times this article has been viewed

\section{Richard R Love \\ The Ohio State University Comprehensive Cancer Center, Columbus, $\mathrm{OH}$, USA}

Correspondence: Richard R Love B402 Starling Loving Hall, 320 W

Tenth Avenue, Columbus,

$\mathrm{OH} 43210$, USA

Tel + I 6I42932268

Fax + I 6I4 2937526

Email richard.love@osumc.edu
Abstract: Cancer is an increasing problem for low- and middle-income countries undergoing an epidemiologic transition from dominantly acute communicable disease to more frequent chronic disease with increased public health successes in the former domain. Progress against cancer in high-income countries has been modest and has come at enormous expense. There are several well-conceived global policy and planning initiatives which, with adequate political will, can favorably impact the growing global cancer challenges. Most financial resources for cancer, however, are spent on diagnosis and management of patients with disease in circumstances where specific knowledge about effective approaches is significantly limited, and the majority of interventions, other than surgery, are not cost-effective in resource-limited countries by global standards. In summary, how to intervene effectively on a global scale for the majority of citizens who develop cancer is poorly defined. In contrast to technology-transfer approaches, markedly increased clinical research activities are more likely to benefit cancer sufferers. In these contexts, a global cancer research initiative is proposed, and mechanisms for realizing such an effort are suggested.

Keywords: breast cancer, research, global, international, low-income, middle-income

\section{Background}

Cancer is an increasing problem in low- and middle-income countries, with high mortality. Cancer kills more people in the world each year than AIDS, tuberculosis, and malaria combined, with cancer accounting for 7-8 million deaths (at least four million in low-income countries), AIDS in about two million deaths, tuberculosis in about 1.3 million deaths, and malaria in about 860,000 deaths. ${ }^{1,2}$ By 2020, the global annual cancer incidence burden will be 20 million (70\% in low- and middle-income countries) and the annual death burden is expected to exceed 10 million. ${ }^{3}$ Each year an increasing proportion of the global cancer burden is occurring in low- and middle-income countries, and, because of their large populations, in the countries of Asia. The incidence rates are increasing, and mortality rates from most cancers remain high in many resource-poor countries. ${ }^{5}$ There are two general differences between higher- and lower-income countries. First, smoking-related cancers are increasing in low-income countries and decreasing in higher-income countries, and the burden of cancer in women is increasing worldwide. Second, the burden of infection-related cancers is much greater in low-income countries than in high-income countries $(25 \%$ versus $6 \%$, respectively). ${ }^{4,5}$

Progress against cancer in high-income countries has been modest and has come at extraordinary expense. Incidence and mortality rates for some cancers have declined in 
selected Western countries, including the US. These salutary trends are consequent to multiple factors, and have occurred mostly in groups with higher socioeconomic status. By the international standards suggested by the World Health Organization (WHO) Commission on Macroeconomics and Health, ${ }^{6}$ few systemic interventions for cancer are significantly cost-effective. Although few proven public health approaches are available, several hold promise, and some preventive interventions will also have beneficial effects on other chronic diseases. For example, antitobacco efforts, which have been successful in high-income countries, can be modified and duplicated with expectations of success in less resource-rich settings. The availability of effective human papilloma virus vaccines holds promise for a major reduction in uterine cervical cancer rates. Reduced smoking is associated with cardiovascular and nonmalignant pulmonary disease benefits. In contrast, many specific cancers are of relatively low incidence, and therapeutic strategies developed in high-income countries are generally very costly.

\section{What do we know and what can be done?}

First, as suggested by a recent Institute of Medicine report, ${ }^{7}$ better cancer control is possible in truly developing countries by appropriate country-specific planning and policies which follow on from already available knowledge.

National Cancer Control plans ${ }^{8,9}$ (as described by the WHO, 2002), and the recent WHO modular reports on Cancer Control: Knowledge into Action, WHO Guide to effective programs; Planning, Prevention, Early Detection, Treatment and Palliative Care (2007-2008). Tobacco control (following the "Framework Convention on Tobacco Control"), hepatitis B virus vaccination (working with the "Global Alliance for Vaccines and Immunization - GAVI"), human papilloma virus vaccination, diet and nutrition (WHO report, and Report of World Cancer Research Fund/ American Institute of Cancer Research, 2007), Centers of Excellence (International Atomic Energy Agency Programme of Action for Cancer Therapy-PACT), palliative care/pain control strategies (as suggested by the WHO and International Narcotics Control Board), surveillance and monitoring (implementation as recommended by the WHO-stepwise approach to chronic disease risk factor surveillance: STEPS; and the Institute of Medicine report). Building on these international recommendations and resources requires leadership, political will, some level of country-specific financial resources, and rigorous evaluative and problem-solving approaches.
Second, emerging data indicate that transmissible/ infectious agents or nutritional components and excesses contribute to, or are responsible for, the majority of global malignancies, explain the very large differences in cancer incidence between different populations, and account for most cancer deaths worldwide. ${ }^{4,5,10}$ Stomach (Helicobacter pylori-associated), liver (hepatitis B-associated) and uterine/ cervical (human papilloma virus-associated) cancers account for the overwhelming majority of the $25 \%$ of cancer deaths currently occurring in low- and middle-income countries. The increasing data associating diet, physical inactivity, and obesity, and increased incidence of several cancers (breast, colorectal, and prostate) have led to the recommendations of the World Cancer Research Fund report. ${ }^{9}$ Despite these circumstances, research resources directed towards understanding and treating these causes are relatively limited in low- and middle-income countries. Specifically, the association between hepatitis B and liver cancer is long-known, and population-effective vaccination programs have been implemented in some countries (eg, Taiwan), but liver cancer still causes approximately 750,000 deaths annually, reflecting an absence of population vaccination coverage. For nutrition-related cancers, such as colorectal and prostate malignancies, the very low rates of these cancers in India, for example, offer important opportunities to investigate protective dietary factors. Globally, the number of etiologic or interventional studies targeted towards these infectious and dietary issues is disproportionally low in comparison with their emerging public health significance.

Third, with respect to cancer diagnosis and systemic treatments other than surgery, which in most countries consumes the vast majority of resources allocated to cancer, knowledge is limited regarding specific effective applications of almost exclusively Western population-derived information in low- and middle-income countries. One critical area is early detection and screening. The incidences of many malignancies vary markedly between countries. Breast cancer incidence in high-income countries is five times greater than in many low-income countries. ${ }^{11}$ This situation alone makes the costs of breast cancer screening and its cost-effectiveness less attractive in a low-incidence, low-income country. Despite expectations that significant downstaging of disease at diagnosis and an associated decrease in case fatality should be associated with well-conducted screening, clinical breast examination, and cervical inspection with acetic acid, the first results of a rigorous Indian clinical trial testing these interventions in 151,538 women after three rounds of screening at two-year intervals, have shown a statistically significant 
downstaging for cervical cancer, with a developing trend for improved case fatality, but limited evidence for downstaging of breast cancer and no improvement in case fatality. ${ }^{12}$ The biology and treatment of cancer exhibit significant differences between populations because of genetic differences in tumors and hosts (which affect metabolism and thus the efficacy and toxicities of systemic treatments), ${ }^{13-15}$ and in other lifestyle and environmental factors. Increasingly, treatments are being designed for specific subgroups of patients with genetically-defined tumor changes. To study these treatments requires casting a wide net to recruit adequate numbers of study subjects in a timely manner. Parenthetically, new evidence suggests that we cannot consider all high-quality technical surgical interventions to be equivalent because host factors at the time of surgery may be associated with long-term outcomes. ${ }^{16}$ We are only beginning to understand the complex interrelationships of mind and body involved in "sickness", and the interactions of cultural and personal representations of illness. Additionally, medical interventions of all kinds vary dramatically in their feasibility, with favorable and unfavorable effects depending on psychosocial and cultural circumstances, particularly poverty. Finally, Western experiences in quality-of-care improvements have highlighted the challenges of defining truly effective interventions. Improvements require changes to be made within complex systems, and unintended consequences often occur. ${ }^{17}$ In these contexts, interventions for cancer beyond surgery in previously unresearched populations (ie, in any other than generally well-off Western Caucasian populations) must be considered of uncertain benefit, and a formative evaluation strategy for application of any intervention in new populations is the safest approach.

The current practice in low- and middle-income countries for wealthy people is that they are offered treatments which are essentially duplicative of perceived state-of-the-art care in high-income countries, with no attention paid to probable biologic/genetic differences in tumors and hosts. Two examples from breast cancer illustrate the hazards of such "one-shoe-fits-all" technology transfer approaches. First, accumulating evidence suggests that there are different alleles of genes critical to the conversion of tamoxifen to its active metabolite, endoxifen. Among Asian populations the more prevalent gene forms lead to limited endoxifen levels and therefore likely limited benefits from this treatment. ${ }^{15,18}$ Second, doxorubicin is an effective chemotherapeutic agent in breast cancer, but again variants in gene alleles are important in its metabolism, leading to greater individual patient exposure to cardiotoxic breakdown products. Emerging evidence indicates that the "bad genes" are more common among Chinese women, in whom permanent iatrogenic heart damage is much more frequent, which changes the risk-benefit calculations for use of this drug. ${ }^{15}$ Third, for the majority of individuals in low- and middle-income countries, cancer care is "catch-as-catch-can" and limited in many ways. It is difficult to support these last two statements with specific and rigorous quantitative data, but after 15 years of on-site visits and patient management discussions with physicians in Vietnam, Philippines, China, Bangladesh, and, less frequently, in several other Asian and African countries, it is probable that they are correct. Fourth, currently defined useful systemic interventions for cancer are generally only widely applied in wealthy countries. The WHO Commission on Macroeconomics and Health has suggested that the cut-off for cost-effective interventions should be that a country's per capita annual income should gain a year of life. ${ }^{6}$ By this standard, only a few current systemic interventions for cancer are cost-effective in most countries.

\section{Summary}

Public health policies are well-defined for important aspects of cancer, but optimal strategies for carrying these out in individual countries are poorly described and evaluated. Research on a global scale regarding the likely transmissible agents and nutritional causes for the majority of cancers and their management is limited. Diagnosis and treatment consume the overwhelming bulk of resources for cancer in any country. Rigorous evidence regarding screening for certain cancers, such as breast and cervical, is lacking in low-income countries. Effective (but often significantly toxic) strategies in selected (genetic) populations have been developed, but these are not cost-effective. For the majority of citizens worldwide, effective, nontoxic, culturally appropriate, and attainable care for cancer has not yet been defined.

\section{Discussion}

A common thread in addressing cancer more effectively throughout the world is the need for research. How to intervene effectively is rarely known. A major increase in all aspects of population-targeted clinical cancer research is required, ie, etiologic, preventive, early detection, diagnosis, treatment, palliative care, and behavioral-psychosocial. In many circumstances what is needed is research specific to local countries.

Viewed globally, the preponderance of current clinical and basic cancer research is in the US and European 
populations. There is a major growing basic science research effort in the East (Hong Kong, Korea, China) and South East Asia (Singapore), and there is promise of similar activity in Saudi Arabia.

In the US, most cancer research has been federally funded, but less than $2 \%$ of the US National Institutes of Health cancer-directed budget is allocated to foreign research and the overwhelming majority of this is to high-income countries, ie, 74 of 78 projects in 2005. The US National Cancer Institute has assisted extensively in training foreign basic science and clinical researchers, and in the development of population cancer registries. There is no doubt that addressing cancer problems in low-income countries is more attainable when there are specific relevant data. Recently, pharmaceutical company funding and studies have dominated the overall clinical interventional research portfolio. These important contributions acknowledge the stark reality that there is little "public health" oncology research. In terms of purchasing dollars, research supported by the US federal government is shrinking.

Financial support for cancer research by nongovernmental organizations is growing, but is still modest compared with federal support, and tends also to be niched in specialized areas and not public health-directed. The truly international, population-based, health-directed cancer research portfolio is embarrassingly small. ${ }^{7}$ Beyond the relatively small, but usually well-targeted, efforts of the International Agency for Research on Cancer (IARC), organizations focusing on international (public health, broad population-benefiting) cancer research are small and few in number, and the Global Research Forum barely considers cancer. The IARC has contributed significantly and usefully to the development of better global cancer data, and meaningfully in developing public health early detection information, but has been resource-limited to follow up these efforts with interventional research. The biggest foundations funding health research are generally confining their attention to long-neglected communicable diseases.

\section{Proposal}

A global cancer research initiative should be launched to develop and facilitate significantly increased numbers of collaborative international research activities and to generate data of significant benefit to individuals with cancer worldwide, particularly to individuals living in countries with limited resources. An international partnership-collaborative group comprising, eg, the US National Cancer Institute, IARC, and European and Asian organizations should be developed to formulate the major goals, vision, and mechanisms for this initiative, and to procure commitments for sustained financial resources to carry out the initiative in rapidly incremental steps. The financial support should come from federal and private sources.

A core staff for the initiative should be recruited and should craft a series of requests for proposals from region-specific planning and organization through to intervention. Also needed are specifications which target low- and middle-income countries, their locally defined priority target areas, and the full spectrum of clinical research areas and funding rules which call for matching funds from all country research partner applicants, with greater levels sought from high-income countries. Rigorous low- and middle-income country clinical research investigator and proposal development, capacity-building training, and partnership/team development programs should be developed under the auspices of organizations such as the American Association for Cancer Research and American Society of Clinical Oncology. The deficit at present lies in mentoring foreign investigators in proposal and research team development.

\section{Conclusion}

Economic development efforts over the last 50 years have been broadly criticized for their limited successes, noting that "planning" rather than "search and research" approaches have dominated. ${ }^{19}$ Much discussion and many activities around addressing the growing cancer burden globally, and the organization and presentations at large clinical meetings, have been characterized by similar planning-technology transfer "solutions". However, when we look at the state-of-the art in what we know about effective interventions in cancer in terms of how these might impact the majority of global citizens, it is clear that a more vigorous research approach is indicated to help low- and middle-income countries develop rigorous data on helpful interventions. The task is great, but if we are to realize the promise of the Declaration of Human Rights, ie, that the fruits of medical science should benefit all mankind, then medical science must more specifically turn its focus to global clinical cancer research.

\section{Acknowledgments}

This research was supported in part by Grant \# CA097375 from the National Institutes of Health and grants from the Breast Cancer Research Foundation and the International Breast Cancer Research Foundation. 


\section{Disclosure}

The author reports no conflict of interest in this work.

\section{References}

1. Parkin DM, Bray F, Ferlay J, Pisani P. Global Cancer Statistics 2002. CA Cancer J Clin. 2005;55:74-108.

2. Kaiser Family Foundation. Fast Facts. Available at: www.globalhealthfacts. org Accessed 2010 February 10.

3. Economist Intelligence Unit. Breakaway: The Global Burden of CancerChallenges and Opportunities. The Economist. 2009.

4. International Agency for Research on Cancer (IARC). In: Stewart BW, Kleihuis P, editors. World Cancer Report. Lyon: International Agency for Research on Cancer; 2003.

5. Rastogi T, Hildesheim A, Sinha R. Opportunities for cancer epidemiology in developing countries. Nat Rev Cancer. 2004;4:909-917.

6. World Health Organization. Macroeconomics and Health: Investing in Health for Economic Development. Report of the Commission on Macroeconomics and Health. Geneva: World Health Organization; 2001. Available from: http://whqlibdoc.who.int/publications/2001/924154550x. pdf. Accessed 2010 April 08.

7. Institute of Medicine. Cancer Control Opportunities in Low and Middle Income Countries. Washington, DC: National Academies Press; 2007.

8. World Health Organization. National Cancer Control Programmes: Policies and Managerial Guidelines. 2nd ed. Geneva: World Health Organisation; 2002. Available from: http://www.who.int/cancer/nccp/ en/. Accessed 2010 April 08.

9. World Cancer Research Fund. Food, Nutrition, Physical Activity and the Prevention of Cancer: A Global Perspective. American Institute for Cancer Research; 2007. Available from: http://www.dietandcancerreport. org/. Accessed 2010 April 08.
10. Reeves GK, Pirie K, Beral V, et al. Cancer incidence and mortality in relation to body mass index in the Million Women study. BMJ. 2007;335:1134.

11. Parkin DM. International variation. Oncogene. 2004;23:6329-6340.

12. Mittra I, Mishra GA, Singh S, et al. A cluster randomized, controlled trial of breast and cervix cancer screening in Mumbai, India: Methodology and interim results after three rounds of screening. Int J Cancer. 2010;126:976-984.

13. Olopade OI, Grushko TA, Nanda R, Huo D. Advances in breast cancer: Pathways to personalized medicine. Clin Cancer Res. 2008;14:7988-7999.

14. Desta Z, Flockhart DA. Germline pharmacogenetics of tamoxifen response: Have we learned enough? J Clin Oncol. 2007;33: 5147-5149.

15. Tan S-H, Lee S-C, Goh B-C, Wong J. Pharmacogenetics in breast cancer therapy. Clin Cancer Res. 2008;14:8027-8041.

16. Badwe RA, Hawaldar RW, Parmar V, et al. Single injection depot progesterone prior to surgery and survival in women with operable breast cancer: A randomized controlled trial. Presented at: 32 nd Annual San Antonio Breast Cancer Symposium, 2009 December 10-13, San Antonio, Texas, USA. Abstr 74. Available from: http://www. abstracts2view.com/sabcs09/index.php. Accessed 2010 April 08.

17. Auerbach AD, Landefeld CS, Shojania, KG. The tension between needing to improve care and knowing how to do it. New Engl J Med. 2007:357:608-613.

18. Schroth W, Goetz MD, Hamann U. Association between CYP2D6 polymorphisms and outcomes among women with early stage breast cancer treated with tamoxifen. JAMA. 2009;302:1429-1436.

19. Easterly W. The White Man's Burden. New York, NY: Penguin; 2007.
Cancer Management and Research

\section{Publish your work in this journal}

Cancer Management and Research is an international, peer-reviewed open access journal focusing on cancer research and the optimal use of preventative and integrated treatment interventions to achieve improved outcomes, enhanced survival and quality of life for the cancer patient The journal welcomes original research, clinical \& epidemiological

\section{Dovepress}

studies, reviews \& evaluations, guidelines, expert opinion \& commentary, case reports \& extended reports. The manuscript management system is completely online and includes a very quick and fair peerreview system, which is all easy to use. Visit http://www.dovepress.com/ testimonials.php to read real quotes from published authors. 